\title{
Review
}

\section{The Role of Elective Neck Dissection during Surgical Salvage for Recurrent Nasopharyngeal Carcinoma}

\author{
Jimmy Yu Wai Chan, Stanley Thian Sze Wong, William Ignace Wei
}

Division of Head and Neck Surgery, Department of Surgery, University of Hong Kong Li Ka Shing Faculty of Medicine, Queen Mary Hospital

Corresponding author: Jimmy Yu Wai Chan; E-mail: chanjyw@gmail.com

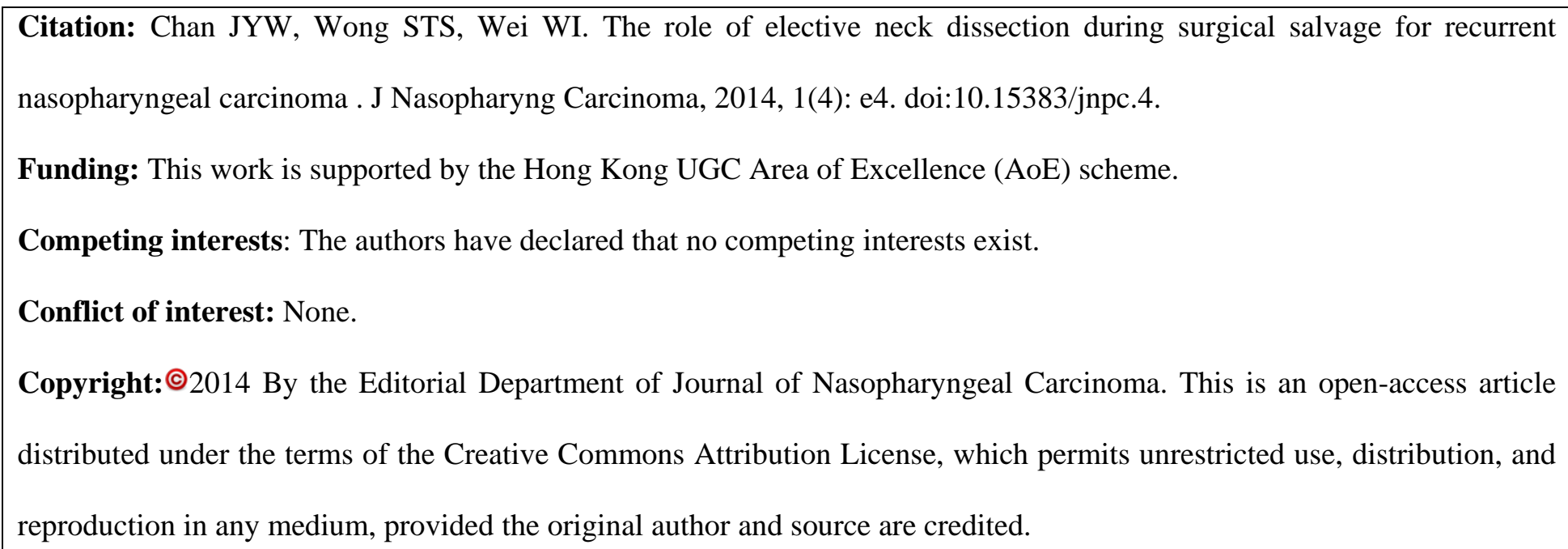

Background: To study the incidence of microscopic tumour deposit in the neck in patients with recurrent nasopharyngeal carcinoma (NPC) and hence, the role of routine elective neck dissection during nasopharyngectomy.

Methods: Retrospective review of the pathology report of the selective neck dissection specimens in patients with recurrent NPC and clinically N0 status.

Results: Between 2000 and 2012, 38 patients presented with recurrent NPC requiring maxillary swing nasopharyngectomy and free flap reconstruction. The initial T-classification of the tumour was $\mathrm{T} 1,15.8 \%$; $\mathrm{T} 2$, $52.6 \%$; and $\mathrm{T} 3,31.6 \%$, and the N-classification was N0, 26.3\%; N1, 47.4\%, N2, 15.8\%; and N3, 10.5\%. Concurrent chemoradiation was given in $57.9 \%$ of the subjects. All patients in the series had complete response after the initial treatment, and the mean time to develop local tumour recurrence was 22.3 months. Among these patients, only 1 (2.6\%) demonstrated microscopic tumour metastasis in 1 lymph node removed during selective neck dissection.

Conclusions:Given the low incidence of microscopic tumour metastasis in patients with recurrent NPC and clinically N0 status, routine elective neck dissection may not be indicated. Further large scale investigation is indicated to address the issue.

Keywords: recurrent nasopharyngeal carcinoma; maxillary swing; elective neck dissection; microscopic tumour deposit

\section{Introduction}

Nasopharyngeal carcinoma (NPC) differs from other head and neck malignancies in its epidemiology, pathology and treatment outcome $^{1,2}$. It is endemic in southern China and Southeast Asia, affecting 10 to 50 per 100,000 populationsper year ${ }^{3}$. The primary treatment is radiotherapy or chemoradiotherapy depending on the stage of disease on presentation ${ }^{4-7}$, whilesurgery is reserved for persistent or recurrent tumours after the combined therapy ${ }^{8}$. 
NPC has the highest preponderance for regional lymph node metastasis among other head and neck malignancies ${ }^{9}$. As a result, the entire neck is empirically irradiated regardless of the stage of disease on presentation ${ }^{10-11}$. While radical neck dissection is often the treatment of choice in regional failures, the need to attend the neck in patients with local tumour recurrence in the nasopharynx is not studied previously. The current investigation will address the role of elective selective neck dissection in locally recurrent NPC.

\section{Patients and Methods}

The study was approved by the Institutional Review Board. Between 2000 and 2012, patients with recurrent NPC, who required nasopharyngectomy and free flap coverage of the parapharyngeal space and skull base, were included in the study. Preoperative investigations included nasoendoscopy and biopsy for histological confirmation and determination of Epstein-Barr virus encoded ribonucleic acid (EBER) status, ultrasound examination of bilateral neck and fine needle aspiration of suspicious lymphadenopathy, and contrast magnetic resonance imaging (MRI) of the nasopharynx and the neck to delineate the local extent of disease and the presence of enlarged lymph nodes in the neck. Patients with cytologically proven metastatic disease in the neck would receive salvage neck dissection at the time of nasopharyngectomy, and they were excluded from the study.

All the recruited patients had large recurrent tumour in the nasopharynx with parapharyngeal extension. They had subsequent nasopharyngectomy performed via the maxillary swing approach. The procedure had been described previously ${ }^{12}$, with a few modifications over the years ${ }^{13}$. Through a Weber-FergusonLongmire incision, osteotomies were made on the anterior wall of maxilla and zygoma and the hard palate. Maxillary tuberosity was separated from the pterygoid plate using a curved osteotome. The maxilla ipsilateral to the tumourwas then swung out to gain access to the tumour.

The nasopharyngeal tumour wasthen resected en-bloc with the pterygoid muscles and the underlying pharyngobasilar fascia to ensure clear margins on the surface of petrosal internal carotid artery. The exposed artery was covered with the free vastus lateralis muscle flap in order to prevent dreaded complications such as carotid blowout. Selective neck dissection, with the removal of ipsilateral level I to III and Va lymph nodes, was performed for all the patients, and suitable recipient vessels were prepared for subsequent microvascular anastomosis. The sternocleidomastoid muscle, the internal jugular vein and the accessory nerve were safeguarded. The maxillary osteocutaneous unit was then returned and the osteotomy sites were fixed with titanium mini plates and screws. A pre-fabricated dental obturator was fitted to ensure accurate dental positioning.

The selective neck dissection specimens were separated into different levels and they were sent separately to the Department of Pathology for histological examination. The specimens were formalin fixed and embedded in paraffin blocks. All the lymph nodes in the selective neck dissection specimens were stained with hematoxylin and eosin (H\&E) for each tissue block and subjected to continuous pathological section. In addition, the detection of tumour was enhanced by in situ hybridization of EBER by Novocastra NCL-EBV-K ISH Kit (Novocastra Laboratories Limited, Balliol Business Park West, Benton Lane, Newcastle, UK). The presence of microscopic tumour deposits were noted and recorded.

All data were entered into the Head and Neck database file and analyzed with Statistical package for social sciences version 18.0 (SPSS, Inc., Chicago IL). A $p$-value of 0.05 or less was considered as significant.

\section{Results}

During the study period, 38patients were included in the study. Majority $(73.7 \%)$ of the patients were male. The median age was 52 years (range 24-62 years). The initial T-classification of the tumour on presentation was $\mathrm{T} 1,15.8 \%$; $\mathrm{T} 2,52.6 \%$; and $\mathrm{T} 3$, $31.6 \%$, and the N-classification was N0, 26.3\%; N1, 47.4\%, N2, $15.8 \%$; and $\mathrm{N} 3,10.5 \%$. Concurrent chemoradiation was given in $57.9 \%$ of the subjects. All patients in the series had complete res 
ponse after the initial treatment, and the mean time to develop local tumor recurrence was 22.3 months. All the tumors had invaded the parapharyngeal space at the time of recurrence.

Pre-operative tumour biopsy yielded undifferentiated carcinoma in all patients. The mean number of lymph nodes removed during selective neck dissection was 24.8 (range, 18 - 30) per neck. Microscopic tumour deposits were identified in only 1 lymph node removed, which was located in the level II. The rest of the lymph nodes examined were negative for malignancy.

\section{Discussion}

The treatment outcome for primary nasopharyngeal carcinoma is improved with the use of intensity-modulated radiotherapy ${ }^{14}$ and concurrent chemoradiation ${ }^{5-7}$. When tumour persists or recurs after the initial therapy, surgeryoffers better local tumour control and survival ${ }^{15-18}$ as well as less post-treatment morbidities than reirradiation

Treatment of recurrent metastatic nodal disease in nasopharyngeal carcinoma requires radical neck dissection ${ }^{19}$. In patients with extensive regional recurrence and extra-capsular spread of cancer cells, extended radical neck dissection with simultaneous insertion of afterloading tubes for early post-operative brachytherapy is effective in improving the treatment outcome ${ }^{20}$. Management of recurrent retropharyngeal lymph node metastasis is more challenging in the diagnosis and treatment, and it can be resected via the maxillary swing approach ${ }^{21}$. The role of prophylactic elective treatment of the neck in patients with locally recurrent NPC, however, is not investigated before. Our previous study on patients with squamous cell carcinoma of the oral cavity showed that selective neck dissection is an effective diagnostic procedure in the staging of $\mathrm{NO}$ neck ${ }^{22}$. It significantly reduces the regional recurrence rate and increases the 5-year disease-free survival ${ }^{23}$.The chance of harboring microscopic tumour deposits in the selective neck dissection specimens of $\mathrm{cN} 0$ patients of carcinoma of the tongue was $22 \%{ }^{24}$.

NPC has the highest preponderance for regional lymph node metastasis among other head and neck malignancies. Studies showed that $85 \%$ of patients with NPC presented with lymphadenopathy ${ }^{25}$, and the most commonly involved regions included retropharyngeal (69\%) and level II lymph nodes (70\%). The outcome of salvage surgery for recurrent nodal disease after radiotherapy, however, has not been satisfactory. The 5-year overall survival and disease-free survival is reported to be as low as $26.03 \%$ and $22.65 \%$, respectively ${ }^{26}$. Therefore, it may be advantageous to better stage the nodal status in at-risk patients who have local tumour recurrence in the nasopharynx, and hopefully, better treatment results can be achieved as observation alone may be dangerous for the irradiated neck. We have previously shown that clinical assessment and fine needle aspiration cytology is insufficient to allow early diagnosis of recurrent nodal metastasis in NPC after irradiation ${ }^{27}$. Therefore, in the current study, we have investigated the incidence of recurrent microscopic nodal metastasis in a group of patients with recurrent NPC who had selective neck dissection performed for the preparation of recipient vessels for microvascular free flap reconstruction after maxillary swing nasopharyngectomy. Our subjects represented a mixed group of patients with various tumour staging before the initial radiotherapy. Majority of them had cervical nodal metastasis at the time of presentation (N1, $47.4 \%, \mathrm{~N} 2,15.8 \%$; and N3, 10.5\%). All of them had complete response after concurrent chemoradiation, only presenting after a mean of 22.3 months with locally advanced tumour recurrence in the nasopharynx invading the parapharyngeal space. By studying the specimen from the selective neck dissection performed in these patients, we showed that the chance of the neck harboring microscopic tumour deposits in patients with cN0 statusat the time of salvage for local failures is only $2.6 \%$. In experienced hands, pre-operative ultrasound and MRI is accurate enough to stage the neck in patients with recurrent NPC after previous radiation. The proposed advantage of limited morbidity after selective neck dissection in patients with squamous cell carcinoma of the head and neck region may not apply in patients with NPC, who have previous routine radical radiotherapy to the bilateral neck with radiation boost to those with regionally advanced disease. Selective neck dissection in these patients may induce further fibrosis and neck stiffness after the procedure, and the functional 
outcome of the spinal accessory nerve may be worse, particularly when removing level IIB lymphatics is mandatory in NPC patients.

However, the limitations of the current investigation, including the retrospective nature of the study design and the small number of subjects recruited, must be taken into account before drawing a definitive conclusion. Further large-scale study is indicated to address the issue adequately.

\section{Conclusions}

Given the low incidence of microscopic tumour deposit in the neck in patients with recurrent NPC and clinically N0 status, routine elective selective neck dissection during nasopharyngectomy may not be justified. Pre-operative assessment by ultrasound and MRI is adequate for accurate staging of the neck in these patients.

\section{Funding Source}

This work is supported by the Hong Kong UGC Area of Excellence (AoE) scheme.

\section{Conflict Of Interest Disclosures}

The authors made no disclosures.

\section{References:}

1. Marks JE, Phillips JL, Menck HR. The National Cancer Data Base report on the relationship of race and national origin to the histology of nasopharyngeal carcinoma. Cancer 1998; 83(3): 582588.

2. Wei WI, Sham JS. Nasopharyngeal carcinoma. Lancet 2005; 365(9476): 2041-2054.

3. Luo J, Chia KS, Chia SE, et al. Secular trends of nasopharyngeal carcinoma incidence in Singapore, Hong Kong and Los Angeles Chinese population, 1973-1997. Eur J Epidemiol 2007; 22: 513-521.

4. Chua DT, Sham JS, Wei WI, et al. The predictive value of the1997 American Joint Committee on Cancer stage classification determining failure patterns in nasopharyngeal carcinoma. Cancer
$2001 ; 92: 2845-2855$.

5. Agulnik M, Siu LL. State-of-the-art management of nasopharyngeal carcinoma: current and future directions. $\mathrm{Br} \mathrm{J}$ Cancer 2005; 92:799-806.

6. Al-Sarraf M, LeBlanc M, Giri PG, et al. Chemoradiotherapy versus radiotherapy in patients with advanced nasopharyngeal cancer: phase III randomized Intergroup study 0099. J Clin Oncol 1998; 16: 1310-1317.

7. Lin JC, Jan JS, Hsu CY, et al. Phase III study of concurrent chemoradiotherapy versus radiotherapy alone for advanced nasopharyngeal carcinoma: positive effect on overall and progression-free survival. J Clin Oncol 2003; 21:631-637

8. Wei WI, Chan YW, Ng WM, et al. Surgical salvage of persistent or recurrent nasopharyngeal carcinoma with maxillary swing approach-critical appraisal after 2 decades. Head Neck $2010 ; 1-8$.

9. Sham JS, Choy D, Wei WI. Nasopharyngeal carcinoma: orderly neck node spread. Int J Radiat Oncol Biol Phys 1990; 19(4): 929933.

10. Lee AW, Sham JS, Poon YF, Ho JH. Treatment of stage I nasopharyngeal carcinoma: analysis of the patterns of relapse and the results of withholding elective neck irradiation. Int $\mathbf{J}$ Radiat Oncol Biol Phys 1989; 17(6): 1183-1190.

11. CA P. Nasopharynx. In: Principles and Practice of Radiation Oncology. 3rd ed. Philadelphia: JB Lippincott; 1998.

12. Wei WI. Cancer of the nasopharynx: functional surgical salvage. World J Surg 2003; 27:844-848.

13. $\mathrm{Ng} \mathrm{RW}$, Wei WI. Elimination of palatal fistula after the maxillary swing procedure. Head Neck 2005; 27:608-612.

14. Lee N, Xia P, Quivey JM, et al. Intensity-modulated radiotherapy in the treatment of nasopharyngeal carcinoma: an update of the UCSF experience. Int J Radiat Oncol Biol Phys 2002; 53: 12-22.

15. Teo PM, Kwan WH, Chan AT, et al. How successful is highdose $(>$ or $=60 \mathrm{~Gy})$ re-irradiation using mainly external beams in salvaging local failures of nasopharyngeal carcinoma? Int J Radiat Oncol Biol Phys 1998; 40:897-913.

16. Fee WE, Robertson JB, GoYnet DR, et al. Long-term survival 
after surgical resection for recurrent nasopharyngeal cancer after radiotherapy failure. Arch Otolaryngol Head Neck Surg 1991; 117:1233-1236.

17. Fee W Jr, Moir M, Choi EC, et al. Nasopharyngectomy for recurrent nasopharyngeal cancer: a 2- to 17-year follow-up. Arch Otolaryngol Head Neck Surg 2002; 128: 280-284.

18. To EW, Lai EC, Cheng JH, et al. Nasopharyngectomy for recurrent nasopharyngeal carcinoma: a review of 31 patients and prognostic factors. Laryngoscope 2002; 112: 1877-882.

19. Wei WI, Mok VW. The management of neck metastases in nasopharyngeal cancer.Curr Opin Otolaryngol Head Neck Surg 2007; 15(2): 99-102.

20. Chan YW, Lee VH, Chow VL, To VS, Wei WI. Extracapsular lymph node spread in recurrent nasopharyngeal carcinoma. Laryngoscope 2011; 121(12): 2576-2580.

21. Chan JY, Chow VL, Wong ST, Wei WI.Surgical salvage for recurrent retropharyngeal lymph node metastasis in nasopharyngeal carcinoma.Head Neck. 2013 Mar 6. doi: 10.1002/hed.23214

22. Yuen AP, Lam KY, Chan AC, Wei WI, Lam LK, Ho WK, Ho
CM. Clinicopathological analysis of elective neck dissection for N0 neck of early oral tongue carcinoma. Am J Surg 1999; 177(1):90-92.

23. Yuen AP, Wei WI, Wong YM, Tang KC. Elective neck dissection versus observation in the treatment of early oral tongue carcinoma. Head Neck 1997; 19(7): 583-588.

24. Yuen AP, Ho CM, Chow TL, et al. Prospective randomized study of selective neck dissection versus observation for N0 neck of early tongue carcinoma. Head Neck 2009; 31(6): 765-772.

25. Ho FC, Tham IW, Earnest A, Lee KM, Lu JJ. Patterns of regional lymph node metastasis of nasopharyngeal carcinoma: a meta-analysis of clinical evidence. BMC Cancer 2012; 12: 98-110. 26. Zhang L, Zhu YX, Wang Y, Huang CP, Wu Y, Ji QH. Salvage surgery for neck residue or recurrence of nasopharyngeal carcinoma: a 10-year experience. Ann Surg Oncol 2011; 18: 233238.

27. Chan JY, Chan RC, Chow VL, To VS, Wei WI.Efficacy of fine-needle aspiration in diagnosing cervical nodal metastasis from nasopharyngeal carcinoma after radiotherapy. Laryngoscope 2013;123(1):134-139. 